\title{
Contribution of the ageing effect to the observed asymmetry of interplanetary magnetic clouds
}

\author{
P. Démoulin ${ }^{1,2}$, S. Dasso ${ }^{3,4}$, V. Lanabere ${ }^{4}$, and M. Janvier ${ }^{5}$ \\ ${ }^{1}$ LESIA, Observatoire de Paris, Université PSL, CNRS, Sorbonne Université, Univ. Paris Diderot, Sorbonne Paris Cité, \\ 5 place Jules Janssen, 92195 Meudon, France \\ e-mail: Pascal.Demoulin@obspm.fr \\ 2 Laboratoire Cogitamus, $13 / 4$ rue Descartes, 75005 Paris, France \\ 3 CONICET, Universidad de Buenos Aires, Instituto de Astronomía y Física del Espacio, CC. 67, Suc. 28, 1428 Buenos Aires, \\ Argentina \\ e-mail: sdasso@iafe.uba.ar \\ ${ }^{4}$ Universidad de Buenos Aires, Facultad de Ciencias Exactas y Naturales, Departamento de Ciencias de la Atmósfera y los Océanos \\ and Departamento de Física, 1428 Buenos Aires, Argentina \\ 5 Institut d'Astrophysique Spatiale, UMR8617, Univ. Paris-Sud-CNRS, Université Paris-Saclay, Bâtiment 121, 91405 Orsay Cedex, \\ France \\ e-mail: miho.janvier@u-psud.fr
}

Received 3 April 2020 / Accepted 8 May 2020

\begin{abstract}
Context. Large magnetic structures are launched away from the Sun during solar eruptions. They are observed as (interplanetary) coronal mass ejections (ICMEs or CMEs) with coronal and heliospheric imagers. A fraction of them are observed in situ as magnetic clouds (MCs). Fitting these structures properly with a model requires a better understanding of their evolution.

Aims. In situ measurements are made locally when the spacecraft trajectory crosses the magnetic configuration. These observations are taken for different elements of plasma and at different times, and are therefore biased by the expansion of the magnetic configuration. This ageing effect means that stronger magnetic fields are measured at the front than at the rear of MCs. This asymmetry is often present in MC data. However, the question is whether the observed asymmetry can be explained quantitatively from the expansion alone.

Methods. Based on self-similar expansion, we derived a method for estimating the expansion rate from the observed plasma velocity. We next corrected the observed magnetic field and the spatial coordinate along the spacecraft trajectory for the ageing effect. This provided corrected data as in the case when the MC internal structure were observed at the same time.

Results. We apply the method to 90 best-observed MCs near Earth (1995-2012). The ageing effect is the main source of the observed magnetic asymmetry for only $28 \%$ of the MCs. After correcting for the ageing effect, the asymmetry is almost symmetrically distributed between MCs with a stronger magnetic field at the front and those at the rear of MCs.

Conclusions. The proposed method can efficiently remove the ageing bias within in situ data of MCs, and more generally, of ICMEs. This allows us to analyse the data with a spatial coordinate, such as in models or remote-sensing observations.
\end{abstract}

Key words. magnetic fields - Sun: coronal mass ejections (CMEs) - Sun: heliosphere

\section{Introduction}

Magnetic emergence and photospheric motions stress the coronal magnetic field, which at some critical point can become unstable. In the frequent cases where the stable overlying magnetic field is not strong enough, a magnetic instability develops, then the plasma and the magnetic field are ejected away from the Sun. These events are called coronal mass ejections (CMEs) and are routinely observed by coronal imagers and coronagraphs (e.g. Sheeley et al. 1985; Schwenn 2006; Howard 2011; Chen 2017). When an ejection is crossed by a spacecraft in interplanetary space, local plasma and magnetic field measurements can be made. A variety of possible criteria has been defined to identify the ejecta signature in the data, and because they are different from the remote-sensing observations of CMEs, the crossed ejections are called interplanetary CMEs (ICMEs, e.g. Cane \& Richardson 2003; Wimmer-Schweingruber et al. 2006; Zurbuchen \& Richardson 2006; Démoulin 2010; Kilpua et al.
2017). When available, the heliospheric imagers can provide a link between a CME observed close to the Sun and an ICMEs observed in situ, showing that ICMEs are the continuation of CMEs away from the Sun (e.g. Harrison et al. 2009; Rouillard 2011; Möstl et al. 2014). An ICME is typically formed by compressed plasma and magnetic field (the sheath) followed by a magnetic ejecta (e.g. Cane et al. 1997; Winslow et al. 2015; Janvier et al. 2019) that is thought to be the continuation of the coronal eruption. When the ejecta has a smooth, coherent, and strong rotation of the magnetic field, and a low proton temperature (compared to the typical solar wind at the same speed), it is classified as a magnetic cloud (MC; e.g. Burlaga et al. 1981; Gosling 1990). Such magnetic structures are typically modelled with twisted magnetic flux tubes, or flux ropes (FRs, e.g. Lepping et al. 1990; Lynch et al. 2003; Dasso 2009; Lanabere et al. 2020).

Coronagraph observations allow us to follow CMEs, showing that they typically expand nearly proportionally with solar 
distance, $D$, away from the low corona (i.e. the size $S$ increase as $S \propto D^{\zeta}$ with $\zeta \approx 1$ ). In contrast, in situ data are typically available only at one solar distance per event because coalignement within a few degrees of two spacecraft observing the same event at different solar distances is rare (Nakwacki et al. 2011; Vršnak et al. 2019; Good et al. 2019). Then, results are typically obtained by analysing large sets of events with the assumption that the statistic is large enough at each distance to erase the individual properties. Such statistical analyses show that the MC radial size increases as a power law of the solar distance (Kumar \& Rust 1996; Bothmer \& Schwenn 1998; Leitner et al. 2007; Gulisano et al. 2010). These results were extended to ICMEs (Liu et al. 2005; Wang et al. 2005). The exponent $\zeta$ is around unity, so the ICME size is nearly proportional to the solar distance, $D$, with variations depending both on the sample and on the distance range analysed, as summarised in Table 1 of Gulisano et al. (2012).

The in situ velocity temporal profiles provide a more direct and systematic way to access the expansion properties within individual events. The proton velocity profile typically decreases almost linearly during the spacecraft passage through the MC (Lepping et al. 2003, 2008; Jian et al. 2008; Gulisano et al. 2010, 2012; Rodriguez et al. 2016; Masías-Meza et al. 2016). This type of velocity profile is expected for a self-similar expansion (Shimazu \& Vandas 2002; Démoulin et al. 2008). Recently, such a self-similar expansion was found to be compatible with the magnetic field profiles obtained for 18 ICMEs that were observed twice with two spacecraft that were nearly radially aligned from the Sun (Good et al. 2019; Vršnak et al. 2019). The physical origin of this evolution is due to the steep decrease of the solar wind total pressure that follows a power law of the solar distance. An approximate pressure equilibrium of the ICME with its surrounding solar wind induces an expansion factor that is also governed by a power law (Démoulin \& Dasso 2009).

A general theoretical framework of the expansion was developed with a hierarchical order from the most general case (ICMEs, anisotropic expansion) to more specific ones (e.g. FRs), and the context and hypothesis were specified at each step (Démoulin et al. 2008). The proton velocity profile measured along the spacecraft trajectory allows us to estimate the expansion rate $\zeta$. These local results globally agree with the statistical results of ICME and MC size evolution versus solar distance (Bothmer \& Schwenn 1998; Gulisano et al. 2010, 2012).

The expansion affects the in situ measurements because the magnetic structure has evolved during the spacecraft crossing. This is known as the ageing effect. For a configuration in expansion, this implies a bias with a stronger magnetic field measured at the front, when the configuration was smaller, than later on, when it is measured at the rear. FR models are typically developed with static configurations, therefore a fit of such models to MC data introduces a bias in the derived parameters. To overcome this, a self-similar expansion is typically supposed. This introduces additional free parameters that are found by including the observed proton velocity in the fitting procedure. The earlier attempts assumed only a 2D expansion orthogonal to the FR axis (Farrugia et al. 1993; Osherovich et al. 1993; Nakwacki et al. 2008). However, this approach is unphysical because the magnetic configuration becomes force imbalanced during the evolution. A more consistent solution is to assume an isotropic 3D expansion. The parameters of the magnetic and velocity models are found by minimising the deviation from the model to both velocity and magnetic data combined within a single function (Shimazu \& Vandas 2002; Dasso et al. 2007; Vandas et al. 2006; Marubashi \& Lepping 2007; Lynnyk \& Vandas 2009).
The improvements of the fit of the magnetic field components by a model in expansion for specific MCs (e.g. Vandas et al. 2015; Marubashi et al. 2017; Vandas \& Romashets 2017) might be an indication that the ageing effect is the main origin of the frequently observed stronger magnetic field found in the front of MCs compared to the values at their rear. However, this is likely to be not generally applicable because this asymmetry is only present in a fraction of MCs, typically faster MCs (Masías-Meza et al. 2016). Furthermore, MCs with a reverse velocity profile, that is, MCs in compression, are also observed (e.g. Gulisano et al. 2010).

The main aim of this paper is to further develop the measurement of MC expansion from in situ data, and to remove the effects of this expansion on the magnetic field data. In Sect. 2 we present the equations describing the self-similar expansion of ICMEs, which relate the expansion factor to the observed velocity. We derive a method for applying these equations to in situ data. In Sect. 3 we derive the expansion profiles of well-observed MCs, and in Sect. 4 we investigate whethere the ageing effect can explain the observed magnetic asymmetry. Next, in Sect. 5 we present a procedure for correcting in situ magnetic data for the ageing effects. Finally, in Sect. 6 we summarise our results and conclude.

\section{Derivation of the expansion rate from the velocity profile}

\subsection{Spatial coordinate and ageing effect}

The in situ measurements provide plasma and magnetic data as a function of time, $t$, because the moving magnetised plasma is crossed by the observing spacecraft. A blob of plasma moving at a velocity $V_{\mathrm{obs}}(t)$ and observed around time $t$ during $\mathrm{d} t$ has a spatial extension

$\mathrm{d} x(t)=V_{\text {obs }}(t) \mathrm{d} t$.

Then, a spatial coordinate along the spacecraft trajectory is derived by integrating the velocity component $V_{\text {obs }}(t)$ observed along the trajectory as

$x(t)=\int_{t_{\text {ref. }}}^{t} V_{\mathrm{obs}}\left(t_{\mathrm{i}}\right) \mathrm{d} t_{\mathrm{i}}$,

where $t_{\text {ref. }}$ is a selected reference time. Compared to the original data, provided as a function of time, rewriting the data as a function of the spatial coordinate $x$ corrects for the effect of the velocity (e.g. a faster blob of plasma appears shorter in the original data).

However, $x(t)$ is not a true spatial coordinate because the crossed structure typically changes its size during the spacecraft crossing. For example, because the MCs shown in Figs. 1a-d are in expansion, the MCs grow in size with time. This global expansion affects each plasma blob, whose size $\mathrm{d} x$ grows with time. Then, $\mathrm{d} x$ is larger when the plasma blob is observed later in the MC. For an MC in contraction, the reverse, that is, a smaller size, is deduced from Eq. (1) when observations are taken closer to the MC rear. This observational bias is called the ageing effect. In situ observations mix time and space because the intrinsic temporal evolution of a plasma blob cannot be observed. Instead, a plasma blob is observed only once, and a different plasma blob is observed at each time. We conclude that $\mathrm{d} x(t)$ in Eq. (1) first needs to be corrected for the ageing effect, then a time integration might provide a true spatial coordinate at a given time across the observed structure (such as in remote-sensing observations). 


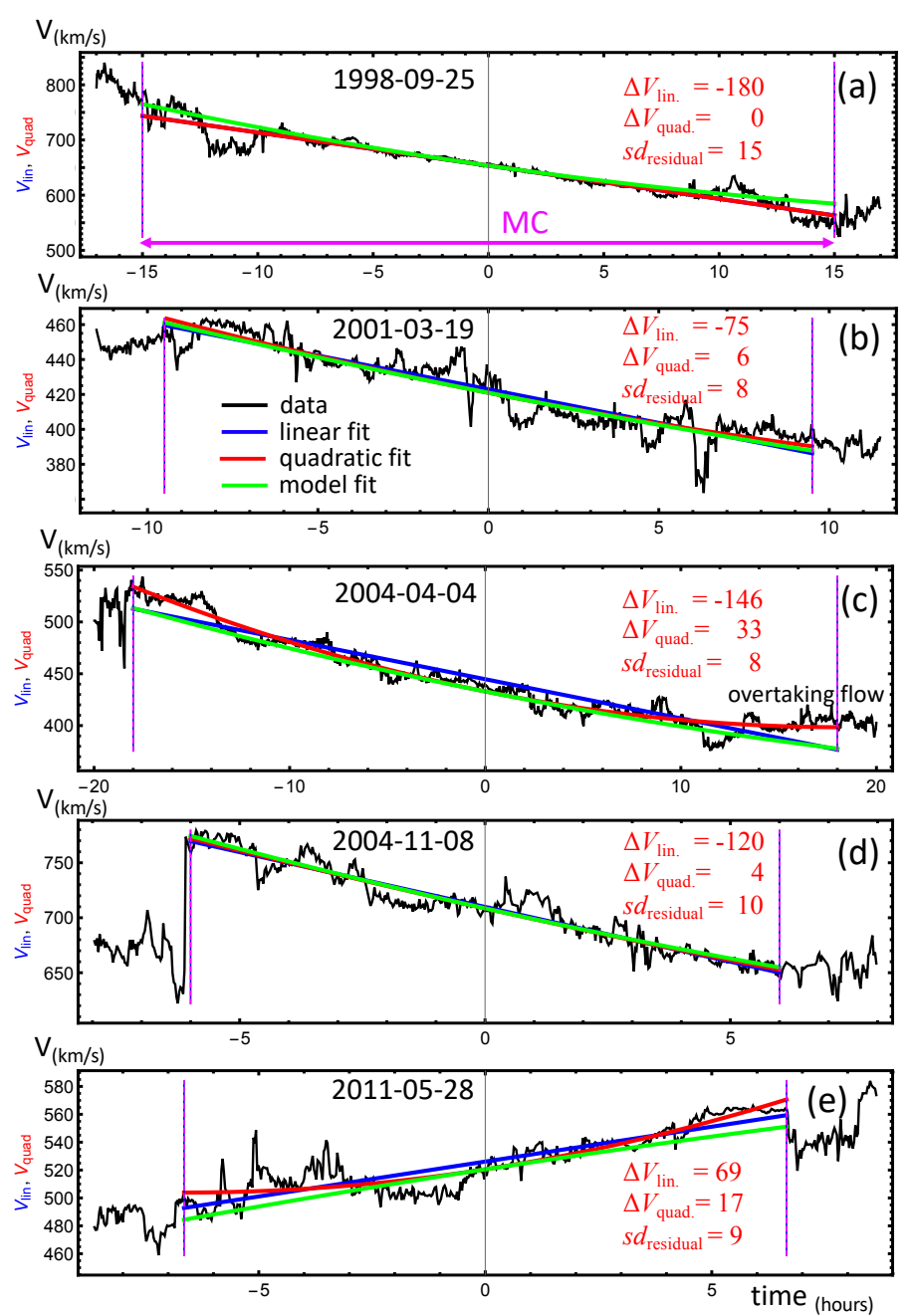

Fig. 1. Examples of velocities measured in situ (black) and fitted results (colours) for five MCs. The labels in the top of the plots indicate the day of the MC front. Linear (in blue) and quadratic (in red) least square fits, Eq. (15), of the observed velocity are over-plotted. The model fit (in green) is an approximation of Eq. (19) (Taylor expansion to second order, so Eq. (21)). The purple vertical lines define the boundaries of the MCs and the range of the velocity fits. Two hours of data are added before and after these boundaries to show the MC context. The time origin is set at MC centre, i.e. $t_{\mathrm{c}}=\left(t_{\text {start }}+t_{\text {end }}\right) / 2$. The main characteristics of the quadratic fit are added in red font; all are in $\mathrm{km} \mathrm{s}^{-1}$ (see Sect. 3.2).

In the following, we derive a method for computing the expansion or compression temporal profile from observed velocities. This is later used to correct for the ageing effect in the observed data (Sect. 5).

\subsection{Global and internal motions}

We present this study in the framework of the 3D expansion theory of ICMEs described by Démoulin et al. (2008), simplifying it to the minimum amount needed to analyse the data within ICMEs that are crossed by a single spacecraft. Such data are the most numerous currently available. The equations derived by Démoulin et al. (2008) for self-similar expansion with possible different rates in three orthogonal directions are only partially constrained by the data of a single spacecraft. Still, because the plasma data constrain the expansion rate along the spacecraft trajectory well, we developed the approach below in order to extract as much information from the data as possible. It is applied to ICME intervals observed in global expansion (or contraction), in particular, to MCs.

We first assumed that the motion of a plasma blob can be described by the sum of a global motion and an internal motion. The global motion is affected by the external forces such as the drag force. The internal motion is driven by internal forces such as the imbalance of total pressure both in the $\mathrm{MC}$ volume and at its boundary with the surrounding medium. We set an axis coordinate $x$ along the spacecraft trajectory, with its origin fixed at the Sun, the location of a plasma blob, labelled $j$, writes

$x_{j}(t)=x_{\text {cent. }}(t)+x_{j \text {, int. }}(t)$,

where $x_{\text {cent. }}(t)$ is the position of the centre of the studied event and $x_{j \text {,int. }}(t)$ is the relative position of blob $j$ with respect to the centre. $x_{\text {cent. }}(t)$ describes the centre of mass motion and $x_{j \text {,int. }}(t)$ the internal evolution. The time derivative of Eq. (3) provides the plasma blob velocity,

$V_{j}(t)=V_{\text {cent. }}(t)+V_{j, \text { int. }}(t)$.

Ideally, the centre is the centre of mass, so that its motion is governed by the result of all external forces applied to the MC. In practice, with in situ data, this mass centre cannot be determined because only a 1D cut is available. The approximation available from in situ data only allows us to set the MC centre at the time $t_{\mathrm{c}}=\left(t_{\text {start }}+t_{\text {end }}\right) / 2$, where $t_{\text {start }}$ and $t_{\text {end }}$ are defined at the MC boundaries.

We next assumed that the internal evolution is a self-similar expansion (or contraction) in the $x$-direction. This implies that the configuration at a time $t$ is a scaled version of the configuration present at another time $t_{\mathrm{c}}$, which writes as

$x_{j, \text { int. }}(t)=x_{j \text {, int. }}\left(t_{\mathrm{c}}\right) f(t)$,

where we set the expansion factor $f\left(t_{\mathrm{c}}\right)=1$ at the reference time $t_{\mathrm{c}}$. In other words, $x_{j}$, int. $\left(t_{\mathrm{c}}\right)$ is a Lagrangian coordinate of the followed plasma blob $j$. Thus, the internal velocity can be expressed as $V_{j \text {,int. }}(t)=\mathrm{d} x_{j \text {, int. }}(t) / \mathrm{d} t=x_{j \text {,int. }}\left(t_{\mathrm{c}}\right) \mathrm{d} f(t) / \mathrm{d} t$. Then, Eq. (4) reads

$V_{j}(t)=V_{\text {cent. }}(t)+x_{j, \text { int. }}\left(t_{\mathrm{c}}\right) \frac{\mathrm{d} f(t)}{\mathrm{d} t}$.

During an MC crossing, the observing spacecraft samples different plasma blobs $j$ at times noted $t_{j}$. The crossing time is typically short, about one day, compared with the timescale of the spacecraft trajectory evolution (about one year for a spacecraft located at $1 \mathrm{au}$ from the Sun). Then, the spacecraft is approximately at a fixed distance from the Sun, called $D_{0}$ below (see more justifications in Sect. 2.1 of Démoulin et al. 2008). Then, including Eq. (5) in Eq. (3) with $t=t_{j}$, Eq. (3) is rewritten as

$x_{j}\left(t_{j}\right)=x_{\text {cent. }}\left(t_{j}\right)+x_{j, \text { int. }}\left(t_{\mathrm{c}}\right) f\left(t_{j}\right)=D_{0}$.

This provides an expression for the unknown position $x_{j \text {, int. }}\left(t_{\mathrm{c}}\right)$ as

$x_{j, \text { int. }}\left(t_{\mathrm{c}}\right)=\left(D_{0}-x_{\text {cent. }}\left(t_{j}\right)\right) / f\left(t_{j}\right)$,

allowing us to eliminate $x_{j \text {, int. }}\left(t_{\mathrm{c}}\right)$ in Eq. (6) written at $t=t_{j}$. It rewrites

$V_{j}\left(t_{j}\right)=V_{\text {cent. }}\left(t_{j}\right)+\left(D_{0}-x_{\text {cent. }}\left(t_{j}\right)\right)\left(\frac{\mathrm{d} \ln f(t)}{\mathrm{d} t}\right)_{t=t_{j}}$. 
Above we explicitly marked the plasma blob with the index $j$ for the derivation of the equations to distinguish between following a given plasma blob $j$ with time $t$, Eqs. (3)-(6), from observing different plasma blobs $j$ at the spacecraft position and at different times $t_{j}$, Eqs. (7)-(9). However, this derivation being achieved, we can simplify the final equation by changing $t_{j}$ to $t$ in Eq. (9), which expresses the continuous observations at the spacecraft with time $t$. This implies that below we keep implicit the reference to observations of different plasma blobs with time and only refer to the observed velocity with the index "obs". In conclusion, the observed velocity in MCs, Eq. (9), is generically modelled as

$V_{\text {obs }}(t)=V_{\text {cent. }}(t)+\left(D_{0}-x_{\text {cent. }}(t)\right) \frac{\mathrm{d} \ln f(t)}{\mathrm{d} t}$.

\subsection{Constraints on the global motion}

The observed proton velocity, $V_{\mathrm{obs}}(t)$, has two contributions: first, the global motion (described by $V_{\text {cent. }}(t)$ ), and second, the expansion (described by $f(t)$ ). These two contributions cannot be separated within the data without additional information.

The frequently observed decreasing profile of $V_{\mathrm{obs}}(t)$ with time in ICMEs could a priori be a consequence of a global deceleration of the ICME when it encounters the spacecraft. However, the magnitude needed for this deceleration would be one to two orders of magnitude larger than the estimated deceleration obtained with three independent methods, as follows.

A first method for estimating the acceleration far from the Sun consists of using observations in quadrature, with a coronagraph imaging the core of the CME while the same event is observed in situ with another spacecraft. These two observations provide an estimate of the mean acceleration from the Sun to the spacecraft, which is an upper bound of the acceleration at the spacecraft crossing because acceleration is stronger close to the Sun (e.g. Rust et al. 2005). A second method consists of statistically deriving the dependence of the ICME velocity with solar distance from in situ data taken at different solar distances (e.g. Liu et al. 2005), and then deducing a typical acceleration. The third method uses observations of spacecraft that are nearly radially aligned from the Sun and observe the same ICME at different solar distances (e.g. Cane et al. 1997; Good et al. 2019; Salman et al. 2020). The velocity measurements at both spacecraft, the distance to the Sun, and the timing at both spacecraft allow us the derivation of two independent estimates of the mean acceleration. These three independent methods show that the acceleration of the ICME centre is generally too weak to explain the profiles of in situ velocities (see Démoulin et al. 2008, for a quantitative analysis that might be updated with the confirmation obtained with the above more recent results).

The above result was confirmed by studies with the imager data of the SoHO and STEREO spacecraft (Liu et al. 2016; Wood et al. 2017; Zhao et al. 2019). Most of the CME deceleration occurs close to the Sun, and the faster events have a stronger deceleration confined closer to the Sun. The imager data are typically consistent with a constant CME velocity for distances from the Sun above $0.3 \mathrm{au}$, and for all cases above $0.6 \mathrm{au}$. The results of the imager data therefore imply that the variations of $V_{\text {cent. }}(t)$ cannot explain the observed in situ velocity variations across ICMEs, and in particular, within MCs, which are typically between 50 and $100 \mathrm{~km} \mathrm{~s}^{-1}$.

\subsection{Expansion rate derived from in situ observations}

In the line of the results of the in situ and imager data on ICMEs and CMEs on their way to $1 \mathrm{au}$, as summarised above, we assumed a constant $\mathrm{MC}$ velocity, $V_{\mathrm{c}}$, of its centre during the spacecraft crossing,

$x_{\text {cent. }}(t)=V_{\mathrm{c}}\left(t-t_{\mathrm{c}}\right)+D_{0}$.

Then, Eq. (10) is rewritten as

$V_{\mathrm{obs}}(t)=V_{\mathrm{c}}-V_{\mathrm{c}}\left(t-t_{\mathrm{c}}\right) \frac{\mathrm{d} \ln f(t)}{\mathrm{d} t}$.

This provides a direct link between the expansion factor $f(t)$ and the observed velocity $V_{\text {obs }}(t)$, which can be rewritten as

$\frac{\mathrm{d} \ln f(t)}{\mathrm{d} t}=-\frac{V_{\mathrm{obs}}(t)-V_{\mathrm{c}}}{V_{\mathrm{c}}\left(t-t_{\mathrm{c}}\right)}$.

Then, Eq. (13) shows that the temporal derivative of the logarithm of $f(t)$ can be calculated with the finite difference of the observed velocity profile computed at $t$ and $t_{\mathrm{c}}$ and normalised with $V_{\mathrm{c}}$. When we assume $V_{\mathrm{obs}}(t)>V_{\mathrm{c}}$ for $t<t_{\mathrm{c}}$, and the reverse for $t>t_{\mathrm{c}}$ (case in expansion), then $\mathrm{d} f(t) / \mathrm{d} t>0$ which implies that the lowest and highest $f(t)$ values are expected at the front and rear MC boundaries, respectively. The same conclusion applies, with an exchange of extrema between boundaries for $V_{\mathrm{obs}}(t)<V_{\mathrm{c}}$ for $t<t_{\mathrm{c}}$, and the reverse for $t>t_{\mathrm{c}}$ (case in compression).

The previous formalism allows us to derive the expansion factor evolution with time, $f(t)$, directly from the observed velocity $V_{\mathrm{obs}}(t)$ by integrating Eq. (13) as

$f(t)=\exp \left(\int_{t_{\mathrm{c}}}^{t} \frac{1-V_{\mathrm{obs}}\left(t_{\mathrm{i}}\right) / V_{\mathrm{c}}}{t_{\mathrm{i}}-t_{\mathrm{c}}} \mathrm{d} t_{\mathrm{i}}\right)$,

with $f\left(t_{\mathrm{c}}\right)$ set to unity (so that $t_{\mathrm{c}}$ is the reference time of the magnetic configuration). The integrand is undetermined for $t_{\mathrm{i}}=t_{\mathrm{c}}$ because both the denominator and the numerator vanish. However, if $V_{\mathrm{obs}}\left(t_{\mathrm{i}}\right)$ is derivable, a first-order Taylor expansion of $V_{\text {obs }}\left(t_{\mathrm{i}}\right)$ around $t_{\mathrm{i}}=t_{\mathrm{c}}$ removes this indetermination. More precisely, this difficulty disappears with $V_{\mathrm{obs}}\left(t_{\mathrm{i}}\right)$ written as $V_{\mathrm{c}}+\left(t_{\mathrm{i}}-\right.$ $\left.t_{\mathrm{c}}\right)\left.\left(\mathrm{d} V / \mathrm{d} t_{\mathrm{i}}\right)\right|_{t_{\mathrm{i}}=t_{\mathrm{c}}}+\left(t_{\mathrm{i}}-t_{\mathrm{c}}\right)^{2} U\left(t_{\mathrm{i}}\right)$, where $U\left(t_{\mathrm{i}}\right)$ contains the remaining expansion and is finite.

The needed smoothness of $V_{\text {obs }}\left(t_{\mathrm{i}}\right)$ in integrating Eq. (14) can be achieved with a local polynomial interpolation around $t_{\mathrm{i}}=t_{\mathrm{c}}$ of $V_{\text {obs }}\left(t_{\mathrm{i}}\right)$ data (e.g. with a spline interpolation). This approach has the advantage of directly incorporating the data in the computation of $f(t)$. However, several phenomena, such as waves and local flows, have contributions in the observed $V_{\text {obs }}(t)$ profile. Such phenomena cannot be modelled with a self-similar expansion hypothesis. We therefore chose to filter out all the velocity contributions at scales smaller than the MC size by first performing a polynomial fit of the observed $V_{\text {obs }}(t)$. Results on MCs show that it is not required to go beyond a polynomial of second order (see Sect. 3.2),

$V_{\text {fit }}(t)=a+b\left(t-t_{\mathrm{c}}\right)+c\left(t-t_{\mathrm{c}}\right)^{2}$,

where $a, b$, and $c$ are the fitted coefficients to the data. The coefficient $a$ is the estimated velocity at the MC centre $\left(t=t_{\mathrm{c}}\right)$, and the coefficients $b$ and $c$ describe the expansion.

After including Eq. (15) in Eq. (14), the integration provides

$f_{\text {fit }}(t)=e^{-\frac{b}{a}\left(t-t_{\mathrm{c}}\right)} e^{-\frac{c}{2 a}\left(t-t_{\mathrm{c}}\right)^{2}}$,

which is a well-behaved function of $t-t_{\mathrm{c}}$. 


\subsection{Expansion rate derived from an expansion model}

In parallel to the previous approach based on fitting the velocity data obtained at a fixed solar distance, $D$, we explore below another approach based on studies that analysed MC sizes that were observed at various solar distances. These statistical studies typically found a power-law dependence of the MC size on solar distance (see Sect. 1). It was shown theoretically that this dependence is expected from the observed power-law decrease of the total plasma pressure of the solar wind with distance (Démoulin \& Dasso 2009). These results imply that $f(t)$ is typically expected to be a power law of solar distance $x_{\text {cent. }}(t)$,

$f_{\text {mod }}(t)=\left(\frac{x_{\text {cent. }}(t)}{D_{0}}\right)^{\zeta}$

with $D_{0}=x_{\text {cent. }}\left(t_{\mathrm{c}}\right)$ being included to have the same normalisation as above $\left(f_{\text {mod }}\left(t_{\mathrm{c}}\right)=1\right)$. The average variation of the total pressure in the solar wind with distance determines a typical $\zeta$ value. However, here we wish to analyse individual MCs where the total pressure in the surrounding solar wind and its variation with distance are not observed. We therefore let $\zeta$ be a free coefficient that is determined from the MC in situ data.

Like in Sect. 2.4, we assumed a constant velocity, $V_{\mathrm{c}}$, for the MC centre during the spacecraft crossing. When we include Eq. (11) in Eq. (17), $f_{\text {mod }}(t)$ is rewritten as

$f_{\text {mod }}(t)=\left(1+\frac{V_{\mathrm{c}}\left(t-t_{\mathrm{c}}\right)}{D_{0}}\right)^{\zeta}$.

When we include this expansion rate in Eq. (10), it provides a model for $V_{\mathrm{obs}}(t)$ as

$V_{\text {mod }}(t)=V_{\mathrm{c}}-\frac{\zeta V_{\mathrm{c}}^{2}\left(t-t_{\mathrm{c}}\right) / D_{0}}{1+V_{\mathrm{c}}\left(t-t_{\mathrm{c}}\right) / D_{0}}$.

When this is applied to the data, $D_{0}$ is the distance of the spacecraft to the Sun, and $t_{\mathrm{c}}$ is the time when the centre of the MC is observed. $V_{\mathrm{c}}$ and $\zeta$ are free parameters that can be determined by a least-squares fit of Eq. (19) to the velocity data.

We next estimated the magnitude of the terms in Eq. (19). To do this, we assumed that the crossed MC is formed of an FR that locally has a cylinder shape of radius $R$ and whose FR axis is inclined by an angle $\gamma$ on the spacecraft trajectory ( $x$ axis). With $t_{\mathrm{B}}$ being the crossing time of one of the FR boundaries, $V_{\mathrm{c}}\left|t_{\mathrm{B}}-t_{\mathrm{c}}\right|$ is lower than $R / \sin (\gamma)$, the equality being obtained in the case of the spacecraft crossing the FR axis. At the Earth orbit distance, typically, $R / D_{0} \approx 0.1$ for MCs, with $R / D_{0}$ rarely reaching $\approx 0.2$ (e.g. Lepping et al. 1990, 2015). Except for MCs that are crossed nearly along their axis or exceptionally large events, this therefore implies that the denominator in Eq. (19) is nearly unity, which implies

$V_{\text {mod }}(t) \approx V_{\mathrm{c}}-\zeta V_{\mathrm{c}}^{2}\left(t-t_{\mathrm{c}}\right) / D_{0}$.

This model describes the nearly linear velocity profile present in unperturbed MCs with the fit of Eq. (20) to data implying $\zeta \approx 1$ for the inner heliosphere (HELIOS spacecraft, Gulisano et al. 2010), to 1 au (Wind and ACE spacecraft, Démoulin et al. 2008), and even to the outer heliosphere up to 5 au (ULYSSES spacecraft, Gulisano et al. 2012).

When we use the same linear approximation in Eq. (18), this implies $f_{\text {mod }}(t) \approx 1+\zeta V_{\mathrm{c}}\left(t-t_{\mathrm{c}}\right) / D_{0}$. Compared to Eq. (20), this implies a direct link between a linear approximation of the velocity and the expansion factor as $f_{\bmod }(t) \approx V_{\mathrm{c}} / V_{\text {mod }}(t)$. This provides a simple estimate of the expansion factor in MCs when the observed velocity is approximately linear (Sect. 3.2). In particular, at the MC boundary, the expansion factor is about $f_{ \pm} \approx 1 \pm \zeta R / D_{0}$, where minus and plus stand for the front and rear boundaries, respectively. Taking $\zeta \approx 1$ and $R / D_{0} \approx 0.1$ provides an expansion factor between 0.9 and 1.1 in typical MCs.

\subsection{Comparison of the methods}

The two analyses in Sects. 2.4 and 2.5 have different hypotheses based on which theory is applied to MC observations. They lead to different expressions for the velocity and the expansion factors. In particular, the first method includes a quadratic fit of velocity observations, and it expresses $f(t)$ with exponentials, Eq. (16), while the second method assumes that the MC size, and therefore $f(t)$, is a power law of solar distance, Eq. (17). This implies different velocity expressions, Eqs. (15) and (19), respectively, with a different input of the data to determine the free parameters.

However, for practical applications to MCs, these two methods are comparable, as follows. The terms in $t-t_{\mathrm{c}}$ contribute less than the leading terms because the radius of the FRs is typically smaller than the solar distance $D_{0}$, as introduced above before Eq. (20). We derive below a Taylor expansion to the second order in $t-t_{\mathrm{c}}$ of the above equations in order to compare the two methods in the context of their application to MCs.

Equation (15) is already a Taylor expansion to the second order of $V_{\text {fit }}(t)$. The same Taylor expansion applied to Eq. (19) is

$V_{\text {mod }}(t) \approx V_{\mathrm{c}}-\zeta V_{\mathrm{c}}^{2} / D_{0}\left(t-t_{\mathrm{c}}\right)+\zeta V_{\mathrm{c}}^{3} / D_{0}^{2}\left(t-t_{\mathrm{c}}\right)^{2}$.

When we compare this equation to Eq. (15), it implies

$c=-a b / D_{0}$

so that the model of Sect. 2.5 can be considered as a particular case of the approach of Sect. 2.4 when it is applied to MCs. The Taylor expansion can also be applied to the expansion factors, Eqs. (16) and (17). With the same velocities, that is, using Eq. (22), the equations are identical, as expected.

\section{Expansion profiles of MCs}

In this section, we first describe the selected MCs, then their velocity profiles. Finally, we apply the above theoretical description to observed MCs in order to derive the expansion factor $f(t)$ from in situ plasma data.

\subsection{Analysed magnetic clouds}

We analysed the data obtained near Earth with the Wind spacecraft, and more precisely, with the Magnetic Field Instrument (MFI) and the Solar Wind Experiment (SWE). The data we used have a temporal cadence of $60 \mathrm{~s}$ for the MFI and $92 \mathrm{~s}$ for the $\mathrm{SWE}^{1}$. The data are provided in the geocentric solar ecliptic (GSE) system of reference.

The 90 MCs included in Lepping's table with the best qualities (1 and 2) were analysed ${ }^{2}$. This table summarises the results of fitting the magnetic data with the Lundquist model as

\footnotetext{
1 They were downloaded from https://cdaweb.sci. gsfc.nasa.gov/pub/data/wind/mfi/mfi_ho and https: //cdaweb.sci.gsfc.nasa.gov/pub/data/wind/swe/swe_h1/, respectively.

2 https://wind.gsfc.nasa.gov/mfi/mag_cloud_S1.html
} 
described by Lepping et al. (1990, 2015), and Lepping \& Wu (2010). These MCs were observed by Wind between 1995-2012.

\subsection{Velocity profiles}

Five examples of MCs are shown in Fig. 1 with the velocity data and three fits. The linear and quadratic fits were derived from a least-squares fit of Eq. (15) to the data within the MC time interval (no expansion model was involved). A power-law model of the expansion with the solar distance, Eq. (18), is also represented. More precisely, a Taylor expansion to second order of the derived velocity, Eq. (21), was used. The central time of the MC was set at $t_{\mathrm{c}}=0$.

The MC examples were selected to represent the variety of magnetic and velocity profiles while still being typical and wellbehaved MCs. Figures 1a-d are examples of MCs in expansion, such as the large majority of MCs, and Fig. 1e is an example of a MC in compression. Of these five examples, two are large MCs (Figs. 1a and c) with a long duration of $\Delta t \approx 27$ and $37 \mathrm{~h}$, respectively, and with an FR radius $\approx 0.2$ au from Lepping's results. The three others, Figs. 1b, d, and e, have shorter durations of $\Delta t \approx 19 \mathrm{~h}, 12 \mathrm{~h}$, and $13 \mathrm{~h}$, respectively, and a smaller FR radius, $0.08,0.09$, and 0.06 au, respectively. Two MCs are fast with $V_{\mathrm{c}} \approx 700 \mathrm{~km} \mathrm{~s}^{-1}$ (Figs. 1a and d), two are slow with $V_{\mathrm{c}} \approx 430 \mathrm{~km} \mathrm{~s}^{-1}$ (Figs. 1b and c), and the last is intermediate with $V_{\mathrm{c}} \approx 520 \mathrm{~km} \mathrm{~s}^{-1}$ (Fig. 1e). Next, except for velocity fluctuations, three MCs have nearly linear velocity profiles (Figs. 1a, b, and d) and two deviate slightly from linearity (Figs. 1c and e).

The velocity profiles in Fig. 1 mainly show a linear variation with time across the MCs, which is expected from a global expansion or compression. Deviations from a linear profile are generally present with velocity fluctuations and when an overtaking flow is present, that is, when a fast solar wind stream impacts the MC rear, for instance in Fig. 1c. In this case, a moderate difference is present between the linear and quadratic fits. The deviation from a linear velocity profile is given by the third term in the right-hand side of Eq. (15). This deviation vanishes at the MC centre $\left(t=t_{\mathrm{c}}\right)$ and is highest at the two MC boundaries, with values equal to $\Delta V_{\text {quad }}=c(\Delta t / 2)^{2}$, while the linear velocity variation across the $\mathrm{MC}$ is $\Delta V_{\text {lin }}=b \Delta t$, where $\Delta t$ is the MC duration, while $b$ and $c$ are the coefficients of Eq. (15) fitted to the velocity data. We also computed the standard deviation of residuals, $s d_{\text {residual }}$, between the velocity fits and the data. The examples shown in Fig. 1 indicate that the linear change in velocity, $\left|\Delta V_{\text {lin }}\right|$, is the dominant effect.

For 12 MCs from a total of 90 , a fast overtaking stream is present at the MC rear. Then, the observed velocity jumps to a moderately higher value close to the MC rear. The time interval with an enhanced velocity is short, shorter than $6 \mathrm{~h}$, and on average, only $2 \mathrm{~h}$. In some MCs, this jump is likely the trace of a shock. The correction for this effect would require applying the technique developed by Wang et al. (2018) to remove the effect of the shock. Here, we instead explore two options: either we fitted the whole MC interval, or we removed the time interval after the shock from the velocity fit, so that the fit was closer to the observed velocity in most of the MCs. A sudden jump in the observed velocity is also present for 5 MCs close to the front boundary, again within a short time interval, shorter than 4 hours, and on average only $2 \mathrm{~h}$.

This shows that removing or leaving these intervals has only a small effect on the results of a small fraction (19\%) of MCs, so that the statistical results below are weakly affected (changing the type of velocity fits is more important).
This method is a different strategy than in our earlier papers, where the aim was to compute the expansion factor $\zeta$ from the part of the velocity profile that was closest to self-similar expansion and therefore mostly linear with time (Démoulin et al. 2008; Gulisano et al. 2010, 2012). In our present work, our aim is instead to include the whole MC duration to compare the results with the three velocity fits, then to correct for the magnetic data from expansion over the whole MC (or at least most of it).

In Fig. 2a the quadratic deviation $\Delta V_{\text {quad }}$ to the linear fit is compared to the linear variation of velocity across the $\mathrm{MC}$, $\Delta V_{\text {lin }}=b \Delta t$. The range of variation of $\Delta V_{\text {lin }}, \approx 300 \mathrm{~km} \mathrm{~s}^{-1}$, and its standard deviation, $\approx 50 \mathrm{~km} \mathrm{~s}^{-1}$, is about twice that of $\Delta V_{\text {quad }}$ $\left(\approx 150\right.$ and $\approx 23 \mathrm{~km} \mathrm{~s}^{-1}$, respectively). The dominance of negative $\Delta V_{\text {lin }}$, with a mean value of $\Delta V_{\text {lin }}=-49 \pm 50 \mathrm{~km} \mathrm{~s}^{-1}$, implies that MCs are typically in expansion, while the quadratic term $\Delta V_{\text {quad }}$ is nearly symmetrically distributed around the origin, with a mean value of $\Delta V_{\text {quad }}=0 \pm 23 \mathrm{~km} \mathrm{~s}^{-1}$. These two terms are not correlated (the Pearson and Spearman correlation coefficients are 0.1 and 0.0 , respectively).

Next, we investigated how far from the regression curve the data points are by computing the standard deviation of the residuals, $s d_{\text {residual }}$ (Figs. $2 \mathrm{~b}-\mathrm{d}$ ). The linear fit typically provides a rather fair fit of the velocity data as $s d_{\text {residual }} \approx 12 \pm 6 \mathrm{~km} \mathrm{~s}^{-1}$, with a maximum of $23 \mathrm{~km} \mathrm{~s}^{-1}$. As expected, with one more free parameter, the quadratic fit has lower residuals, $s d_{\text {residual }} \approx$ $9 \pm 5 \mathrm{~km} \mathrm{~s}^{-1}$.

Next, the model fit was derived from the quadratic fit with the coefficient $c$ imposed by Eq. (22). This significantly increased the deviation from the data $\left(s d_{\text {residual }} \approx 16 \pm 12 \mathrm{~km} \mathrm{~s}^{-1}\right)$. This implies that the global expansion, modelled with a power-law function of solar distance (Sect. 2.5), does not provide the quadratic term present in the observations. The weak quadratic term of this expansion model is indeed expected to be masked by the term implied by the interactions with the surrounding medium, that is, compression from the sheath and/or from an overtaking stream (like in the examples of Figs. 1a, c, and e).

We conclude that the velocity fits, especially the quadratic fit, provide a fair representation of the data. In particular, the residuals typically remain smaller than the global velocity variations $\Delta V_{\text {lin }}$ and $\Delta V_{\text {quad }}$ (Fig. 2). Finally, we note that the examples of Fig. 1 show typical values of $s d_{\text {residual }}$, therefore they provide fair examples of the typical fluctuations present in the MCs we studied.

\subsection{Expansion factors}

The expansion factor is derived from Eq. (16) with the coefficients $a, b$, and $c$ provided by the velocity fits. The central time of the MC, $t_{\mathrm{c}}$, is selected for the reference time, $f_{\mathrm{fit}}\left(t_{\mathrm{c}}\right)=1$. The five selected MCs have nearly linear variation of $f(t)$, with only small deviations between the linear and quadratic fits, as shown in Fig. 3. The small contribution of the quadratic term in $f(t)$ partly arises because its contribution in the velocity profile is lower than that of the linear term (Fig. 2a). Next, the quadratic term of $f(t)$ in Eq. (16) is divided by a factor 2 compared to $V_{\text {fit }}(t)$ in Eq. (15) ( $c$ is divided by 2 in Eq. (16) as a result of the integration within Eq. (14)). This further decreases its contribution in $f_{\text {fit }}(t)$ compared to $V_{\text {fit }}(t)$. Finally, the variation in velocity across an MC is typically smaller than its central (or mean) velocity, so that Eq. (16) can be expanded to the first order in $t-t_{\mathrm{c}}$ to a good approximation, even better than $V_{\text {fit }}(t)$ (lower quadratic term), providing a nearly linear profile for $f(t)$, as shown in Fig. 3. 


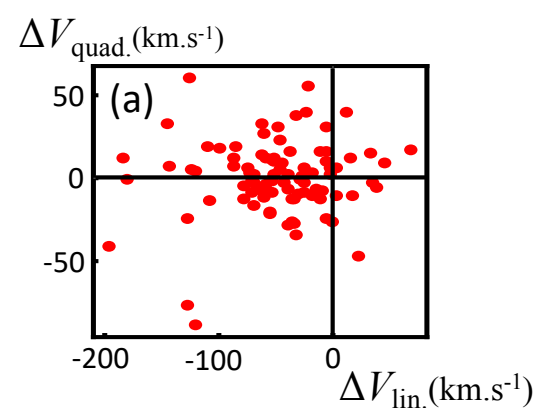

quadratic $V$ fit

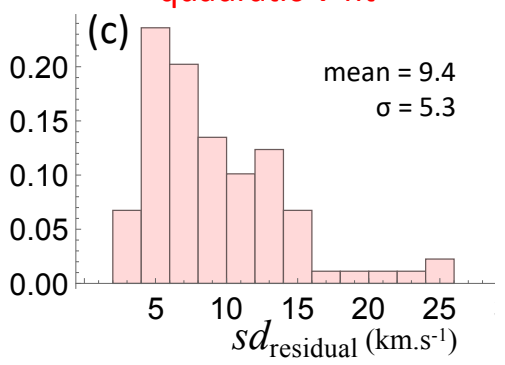

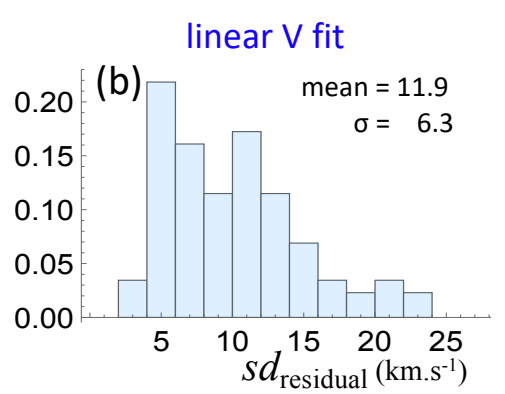

model $V$ fit

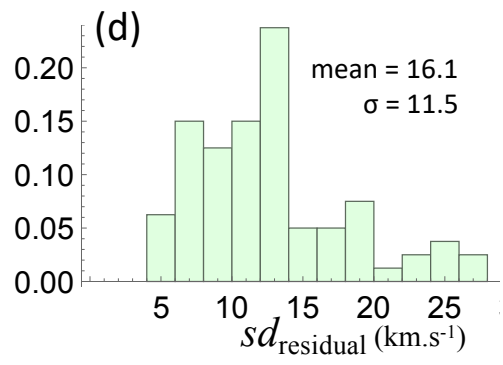

Fig. 2. Fit properties for the $90 \mathrm{MCs}$ we studied. (a) Maximum contribution across the MCs of the quadratic term, $\Delta V_{\text {quad }}=c(\Delta t / 2)^{2}$ as a function of the linear variation of velocity $\Delta V_{\text {lin }}=b \Delta t . \Delta t$ is the MC duration, and $b$ and $c$ are the coefficients of Eq. (15) fitted to the velocity data. $(b-d)$ Histograms of the standard deviation of residuals between the velocity fits and the data. The linear, quadratic, and model fits are plotted in light blue, red, and green, respectively.
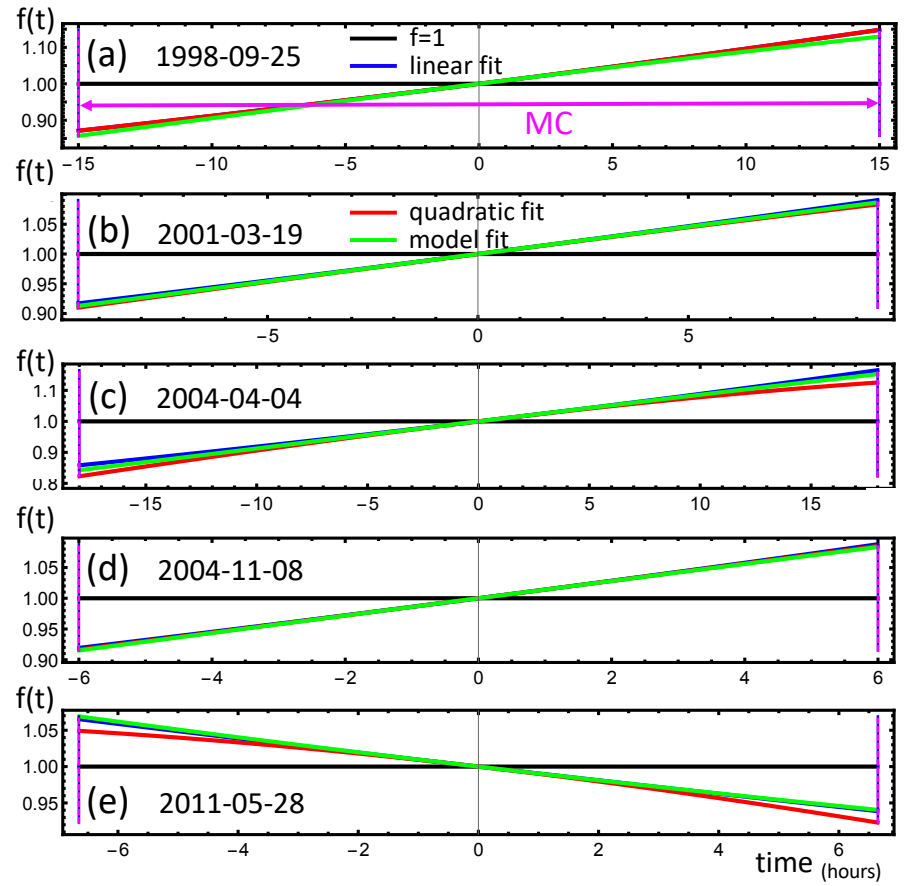

Fig. 3. Expanding factors $f_{\text {fit }}(t)$, Eq. (16), corresponding to the MC velocities shown in Fig. 1. $f_{\text {fit }}(t)$ are derived from linear, quadratic, and model fits of the observed velocities. The temporal MC centre, located at $t=0$, is the reference time for correcting for the ageing effects (i.e. $f(0)=1)$.

We furthermore note that if the velocity data were directly implemented in Eq. (14) to compute $f(t)$, the time integration would smooth the velocity fluctuations efficiently away from the MC centre. We therefore conclude that the expansion factor away from the MC centre would be close to the above estimations with data fits and would be dominated by the linear term when the velocity profile is dominantly linear within an MC, which is typical.

The importance of the $\mathrm{MC}$ expansion during the spacecraft crossing is shown in Fig. 4 with the expansion factors found at
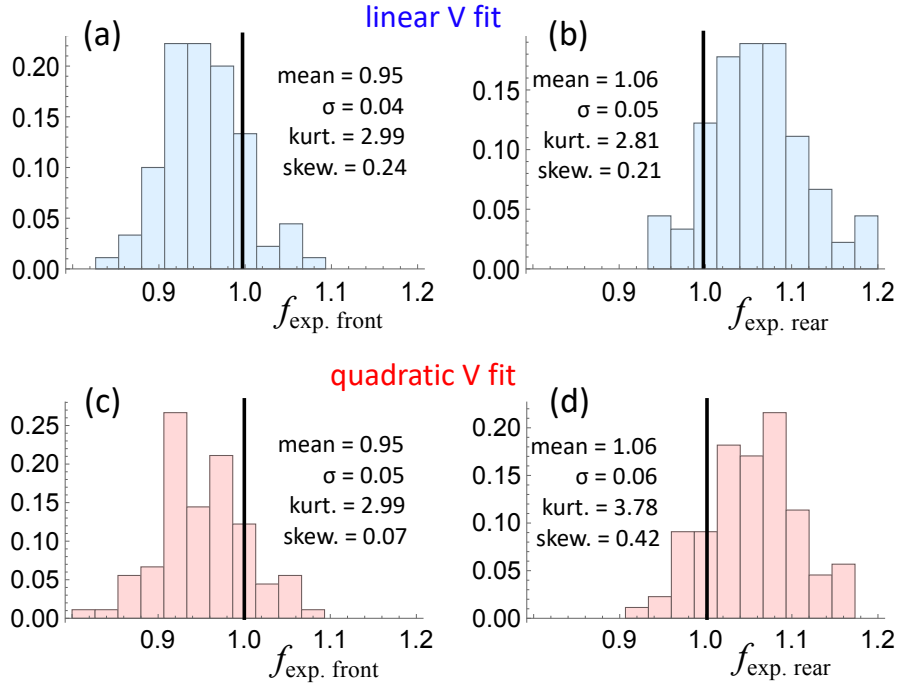

Fig. 4. Histograms of the expansion factors $f_{\text {fit }}$, Eq. (16), computed ( $a$ and $c$ ) at the front, and ( $b$ and $d$ ) at the rear MC boundaries. They are the extreme values of $f(t)$ across MCs. $f_{\text {fit }}$ values are derived from linear (top) and quadratic (bottom) fits of the observed velocities (similar results are obtained with the model fit).

the front and rear boundaries. They are extreme values within each MC (see the analysis after Eq. (13) and Fig. 3). All other parameters being equal, more extreme values of $f_{\text {fit }}$ values, that is, away from unity, are expected at the periphery of the longer duration MCs, as is obtained for the MCs in Figs. 3a and c (see Démoulin et al. 2008, for a further theoretical analysis).

The expansion factors are spread in the interval $[0.8,1.1]$ for the front values and $[0.9,1.25]$ for the rear values. This shows that the expansion weakly transforms the MCs during their observing time, as expected from the typical values $(0.9$ and 1.1) derived at the end of Sect. 2.5. The histograms with linear and quadratic fits are slightly different, indicating that a fraction of MCs have some differences in $f_{\text {exp.front }}$ and $f_{\text {exp.rear }}$, as in the examples of Figs. 3c and e. However, the statistical parameters of $f_{\text {exp. }}$ distributions are similar, and globally, there are only 
weak differences in the expansion factors that are computed with linear and quadratic fits.

A few MCs have $f_{\text {exp.front }}>1$ or $f_{\text {exp.rear }}<1$, indicating a compression (e.g. $11 \%$ have $f_{\text {exp.front }}>1$ and $18 \%$ have $f_{\text {exp.rear }}<1$ with a quadratic velocity fit). Still, for most MCs, the expansion factor is typically below (above) unity at the front (rear) boundary, showing that MCs are typically in expansion.

\section{Magnetic asymmetry and expansion rate of MCs}

\subsection{Magnetic asymmetry}

The asymmetry of the magnetic field intensity in MCs was quantified by Janvier et al. (2019) and Lanabere et al. (2020). They used the coefficient $c_{B, t}$, which is defined as

$c_{B, t}=\int_{t_{\text {start }}}^{t_{\text {end }}} \frac{t-t_{\mathrm{c}}}{t_{\text {end }}-t_{\text {start }}} B(t) \mathrm{d} t / \int_{t_{\text {start }}}^{t_{\text {end }}} B(t) \mathrm{d} t$,

with the central time $t_{\mathrm{c}}=\left(t_{\mathrm{start}}+t_{\mathrm{end}}\right) / 2$.

We quantified the $B(x)$ profile asymmetry in a similar way by defining $c_{B, x}$ like $c_{B, t}$, but with the integration done on the spatial coordinate $x$,

$c_{B, x}=\int_{x_{\text {start }}}^{x_{\text {end }}} \frac{x-x_{\mathrm{c}}}{x_{\text {end }}-x_{\text {start }}} B(x) \mathrm{d} x / \int_{x_{\text {start }}}^{x_{\text {end }}} B(x) \mathrm{d} x$,

with the central position $x_{\mathrm{c}}=\left(x_{\text {start }}+x_{\text {end }}\right) / 2$. The normalisation by the MC size $\left(x_{\text {end }}-x_{\text {start }}\right)$ at the denominator implies that $c_{B, x}$, like $c_{B, t}$, is dimensionless. $x$ is computed with Eq. (2) and could be further corrected for the ageing effect (with $x$ replaced by $x^{\prime}$, see Sect. 5.1).

When the $B(x)$ profile is symmetric around $x_{\mathrm{c}}, c_{B, x}=0$. $\left|c_{B, x}\right|$ increases as the asymmetry of the profile increases, with $c_{B, x}$ negative when $B$ is stronger before $x_{\mathrm{c}}$, and positive when the field is more concentrated towards the MC rear. $c_{B, x}$ is the difference of two oppositely signed quantities (for $x<x_{\mathrm{c}}$ and $x>x_{\mathrm{c}}$ ), moreover, it includes the normalisations by the spatial size and the full integral of $B$. All these contribute to define low values of $\left|c_{B, x}\right|$ while the asymmetry of $B(x)$ in a studied MC may appear very strong, as is shown in plots of $B(x)$, such as in Fig. 5.

In the expected range of $c_{B, x}$ values can be computed with the simple model where $B(x)$ decreases linearly by $\Delta B \geq 0$ across the $\mathrm{MC}$ with a central field $B_{\mathrm{c}}$. Including this model in Eq. (24), we derive $c_{B, x}=-\Delta B /\left(12 B_{\mathrm{c}}\right)$. An extreme case is when $B=0$ at the rear boundary, which corresponds to $B=2 B_{\mathrm{c}}$ at the front boundary, then $\Delta B=2 B_{\mathrm{c}}$. For this strong $B$ asymmetry, $c_{B, x}=-0.167$. The MC data shown in Fig. $5 \mathrm{~d}$ are comparable to this profile, but are slightly less asymmetric. When we instead include $\Delta B=B_{\mathrm{c}}$ in the model, that is, a magnetic field that decreases from $3 B_{\mathrm{c}} / 2$ at the front to $B_{\mathrm{c}} / 2$ at the rear, $c_{B, x} \approx-0.083$. This result is similar to the MC data shown in Fig. 5a.

The coefficients $c_{B, x}$ and $c_{B, t}$ can be compared by performing a Taylor expansion of $V_{\mathrm{obs}}(t)$ and $B(t)$. This allows us to analytically compute the integrals in Eqs. (23) and (24). For our purpose, linear expansions of $V_{\mathrm{obs}}(t)$ and $B(t)$ are sufficient within MCs. This implies

$c_{B, t}=\mathrm{d} b / 12$,

$c_{B, x}=c_{B, t}\left(1-\mathrm{d} v^{2} / 20\right) /(1+\mathrm{d} b \mathrm{~d} v / 12)$,

where $\mathrm{d} b=\Delta B / B_{\mathrm{c}}$ and $\mathrm{d} v=\Delta V_{\text {obs }} / V_{\mathrm{c}}$ are the relative changes across the full MC. In the analysed MCs, we have $|\mathrm{d} b| \lesssim 1$ and $|\mathrm{d} v| \lesssim 0.3$ (e.g. see $\Delta V_{\text {lin }}$ in Fig. 2). Within these limits, Eq. (26)
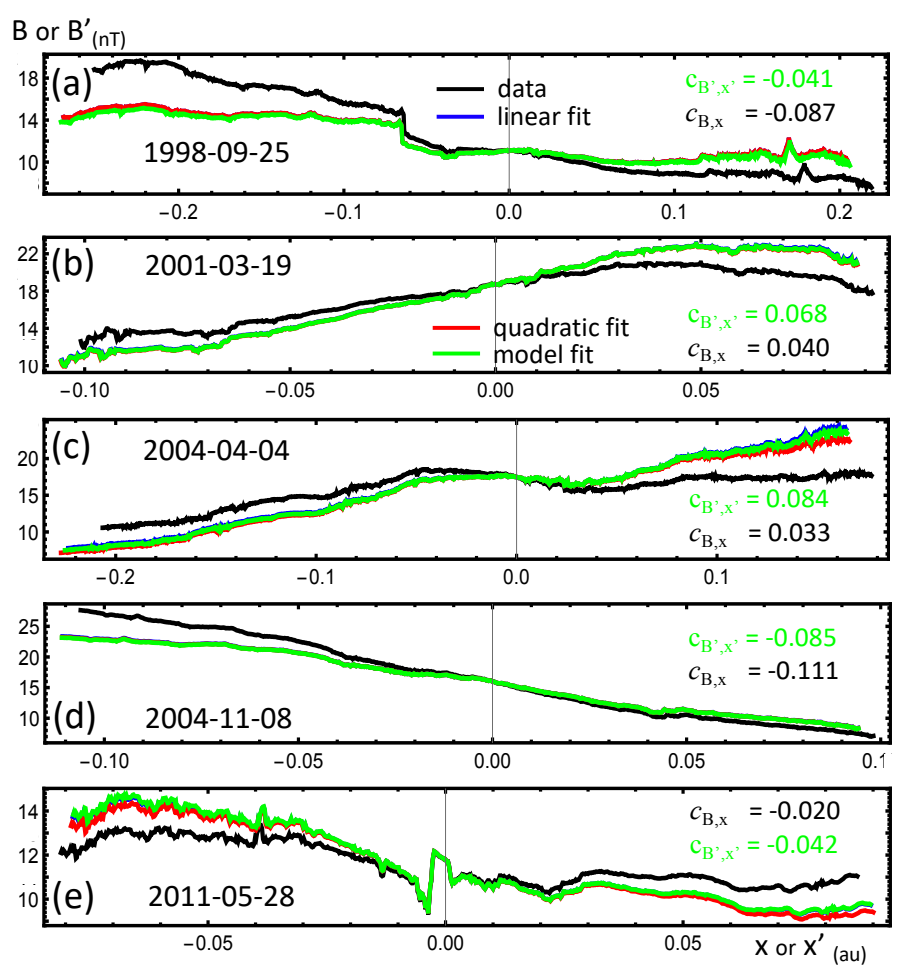

Fig. 5. Magnetic field magnitude, $B$, across the same MCs as shown in Figs. 1 and 3. The black lines show the original data vs. the spatial coordinate $x$, Eq. (2), along the spacecraft trajectory. The coloured curves show the results of removing the ageing effect both on $B$ and $x$ with the expansion factors shown in Fig. 3, so that they show $B^{\prime}\left(x^{\prime}\right)$ (Eqs. (27) and (28)). The plotting order is black, blue, red, and green lines, and the model fit curves mostly mask the underlying results obtained with other fits. Two magnetic asymmetry values, $c_{B, x}$ and $c_{B^{\prime}, x^{\prime}}$, are reported on the right side of each panel (with the same colour convention).

implies $c_{B, x} \approx c_{B, t}$ with the largest difference coming from the denominator contribution. The computation can be extended to more terms, but the low values of $|\mathrm{d} v|$ imply even smaller contributions.

In summary, $\left|c_{B, x}\right|$ increases with the magnetic asymmetry, and a large asymmetry is marked with $\left|c_{B, x}\right|$ around or larger than 0.1. $c_{B, x}$ is similar to $c_{B, t}$ for MCs, and $c_{B, x}$ includes the effect of ageing and the intrinsic spatial asymmetries. $c_{B, x}<0$ marks a $B$ field that is stronger in the MC front, and $c_{B, x}>0$ marks a $B$ field that is stronger at the rear.

\subsection{Ageing effect on the magnetic asymmetry}

We report in Fig. 6 the values of $f(t)$ found at MC boundaries with the asymmetry of $B$ computed from Eq. (24). This confirms the results of histograms (Fig. 4) that linear and quadratic fits show moderate differences in expansion factors, as shown with the typical small shift in ordinate between blue and red pairs of points (only a few cases have a difference of $f_{\text {exp.front }}$ and $f_{\text {exp.rear }}$ between 0.05 and 0.1 ).

Next, we explored the expected ageing effect on the observed $B$ field. We recall that $f_{\text {exp.front }}<1$, and $f_{\text {exp.rear }}>1$ is the expected signature of the expansion, and the opposite inequalities are for contraction. Next, we considered a hypothetical intrinsic symmetric MC $\left(c_{B, x}=0\right)$ as was considered in Démoulin et al. (2008) with an FR model. The inclusion of expansion implies $c_{B, x}<0$, while compression implies $c_{B, x}>0$ on the data simulating a spacecraft crossing the 

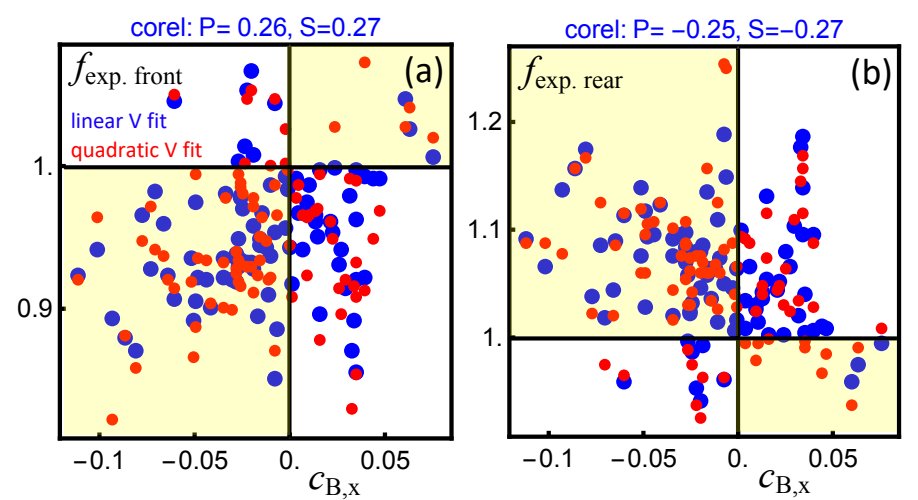

Fig. 6. Expansion factors $(a)$ at the front and $(b)$ at the rear MC boundaries as a function of the magnetic asymmetry coefficient $c_{B, x}$, i.e. Eq. (24) applied to the magnetic data. The results of linear (blue) and quadratic (red) fits for a given MC are shown with two nearby points located on the same vertical line. The model fit derived from Eq. (21) (in green in earlier figures) is omitted because the results are similar. The Pearson (P) and Spearman (S) correlation coefficients are reported at the top of each panel for linear velocity fits. The ageing effect alone is expected to create a magnetic asymmetry that is correlated with the expansion factors, so that for asymmetry due to ageing, data points are expected to be included in the yellow regions.

FR in the conditions of observations (i.e. including the ageing effect).

With a significant ageing effect on the magnetic asymmetry, we would expect a clustering of the data points in the yellow regions in Fig. 6. Even more, higher $\left|c_{B, x}\right|$ values are expected when expansion or compression is stronger. No such expectations are present in Fig. 6 because the points are dispersed, both globally and even inside the yellow regions. We conclude that the results of Fig. 6 imply that the ageing effect is not the main cause of the magnetic asymmetry observed in MCs. Still, because moderate correlation coefficients are found $(\approx \pm 0.26$, top of Fig. 6), the ageing effect is expected to affect the asymmetry moderately.

\section{Removing the ageing effects within MCs}

The main aim of this section is to remove the bias produced by coupling space and time when single-point observations are made on different elements of plasma at different times (ageing effect). Then, the aim is to provide data as if they were obtained at the same time within the $1 \mathrm{D}$ cut of the observed MC.

\subsection{Method for correcting for the ageing effects}

The estimation of $f(t)$ presented above allows us to correct the observations for the ageing effect within the hypothesis of a self-similar expansion. More precisely, based on the information derived from the observed velocity profile, we correct the observed magnetic profile $\boldsymbol{B}(t)$ in field strength, then we transform it into $\boldsymbol{B}\left(x^{\prime}\right)$ as if the observations had been obtained at the same time across the MC. The corrections are both on the spatial scale and on the field strength as follows.

The correction on the spatial scale, by $1 / f(t)$, is to be applied on the elementary length $\mathrm{d} x(t)$, Eq. (1), observed at time $t$ (and not on $x(t)$, Eq. (2), which cumulates the elementary length observed at different times). This defines the spatial coordinate $x^{\prime}$ as if the full $\mathrm{MC}$ had been observed at the time $t_{\mathrm{c}}$ with

$x^{\prime}(t)=\int_{t_{\text {ref. }}}^{t} \frac{V_{\text {obs }}\left(t_{\mathrm{i}}\right)}{f\left(t_{\mathrm{i}}\right)} \mathrm{d} t_{\mathrm{i}}$.

Because $f(t)$ is mostly a linear function (Fig. 3), this implies an antisymmetric increase (decrease) of the front (rear) extension of the MCs in expansion, as shown in Figs. 5a-d (same MC examples as in Figs. 1 and 3). A reverse effect is present for MCs in compression (e.g. Fig. 5e). This transformation of $x$ into $x^{\prime}$ almost conserves the MC size because the front is extended by nearly the same amount as the rear is contracted (to second order in the MC duration $\delta t$ ).

The correction of the magnetic field needs another hypothesis because the data only allow us to derive the expansion along the spacecraft trajectory. The 3D expansion can be derived from observations of the same MC by two spacecraft located at different radial distance from the Sun, as was performed for one MC by Nakwacki et al. (2011). The results are similar to isotropic self-similar expansion. A nearly isotropic expansion is also generically expected with an expansion driven by the adjustment towards total pressure equilibrium between the MC and its surroundings when the MC moves away from the Sun (Démoulin $\&$ Dasso 2009). Furthermore, the analysis of in situ observations indicates an expansion rate, $\zeta$, in the radial direction (away from the Sun) that is on average comparable to the expected orthoradial expansion rate, $\approx 1$. This implies spatial scales in three orthogonal directions that are almost proportional to the solar distance (Démoulin et al. 2008; Gulisano et al. 2010, 2012).

Following the results above on expansion, we assumed an isotropic self-similar expansion to correct for the magnetic field. This evolution can be included in the more general context of ideal MHD for size rescaling of an initial MHD state $\boldsymbol{B}_{0}(\boldsymbol{r})$ as $\boldsymbol{B}(f \boldsymbol{r})=f^{-2} \boldsymbol{B}_{0}(\boldsymbol{r})$. This rescaling is the consequence of flux conservation at the level of each elementary fluid bubble. An isotropic expansion (compression) of the fluid between two states means that all spatial scales increase (decrease) as $\boldsymbol{r} \rightarrow f \boldsymbol{r}$. This implies that the magnetic field is modified to $\boldsymbol{B}_{0}(\boldsymbol{r}) \rightarrow$ $f^{-2} \boldsymbol{B}_{0}(\boldsymbol{r})$ to conserve the magnetic flux $\left(B_{0} \mathrm{~d} r^{2}=f^{-2} B_{0} f^{2} \mathrm{~d} r^{2}\right)$. Here, the rescaling $f(t)$ is a function of time (because the MC evolves when the spacecraft crosses it). Then, in order to remove the ageing effect, all magnetic field components were rescaled by multiplying them with $f^{2}(t)$. Including the transformation of $t$ into $x^{\prime}$, Eq. (27), this provides

$\boldsymbol{B}^{\prime}\left(x^{\prime}\right)=\boldsymbol{B}(t) f^{2}(t)$.

This transformation ensures magnetic flux conservation.

The isotropic expansion can be a basic hypothesis for MCs that are deformed during their propagation in the solar wind, as was shown in some numerical simulations (e.g. Cargill \& Schmidt 2002; Manchester et al. 2004; Lugaz et al. 2005; Xiong et al. 2006). However, the shape of FRs is a consequence of the full evolution from the Sun to the spacecraft, while the expansion correction is here only applied during the MC crossing, therefore our hypothesis of isotropic self-similar expansion is expected to be a good approximation during the spacecraft crossing. Next, while equations with a different expansion rate in three orthogonal directions have been developed (Démoulin et al. 2008), the typical observations by one spacecraft do not allow us to constrain the three expansion factors. Finally, in view of the moderate corrections introduced by removing isotropic expansion effects, including 3D expansion effects is expected to be a correction at the next order of magnitude. 


\subsection{Magnetic asymmetry corrected for the ageing effect}

The spatial magnetic profiles derived directly from the data and those corrected for the ageing effect are compared in Fig. 5 for the same MC examples as are shown in Figs. 1 and 3. The MC examples have moderate to large $B$ asymmetries that are of both signs. We first describe the results for the MC examples with the two strongest ageing effects (Figs. 3a,c). The maximum correction for ageing is about $4 \mathrm{nT}$ for $B \approx 19 \mathrm{nT}$ (Fig. 5a) and $6 \mathrm{nT}$ for $B \approx 17 \mathrm{nT}$ (Fig. $5 \mathrm{c}$ ), that is, at most, a correction of $35 \%$. The correction reduces the asymmetry of $B$ between the front and the rear of the MC in Fig. 5a, as summarised by the significant reduction of $\left|c_{B, x}\right|=0.087$ to $\left|c_{B^{\prime}, x^{\prime}}\right|=0.042$. The correction is smaller in Fig. 5d $\left(\left|c_{B, x}\right|\right.$ is reduced by 25\%). This is especially true at the MC rear, where the correction of $x$ shifts the nearly linear $B$ profile towards the MC front, so that $B^{\prime}\left(x^{\prime}\right)$ is similar to $B(x)$ in this rear region. The same effect is present in panel a, but there the ageing removal is stronger, so that $B^{\prime}\left(x^{\prime}\right)$ and $B(x)$ are farther away.

Conversely, an increase in asymmetry is present from $c_{B, x}$ to $c_{B^{\prime}, x^{\prime}}$ with a factor 1.7 and 2.7 in Figs. $5 \mathrm{~b}$ and c, respectively. This is a direct consequence of MCs in expansion because the already weaker $B$ at the front is even weaker after the correction, while the opposite holds at the MC rear. As expected for MCs in compression with a stronger $B$ in their front, as shown in Fig. 5e, the asymmetry is also increased after the correction $\left(\left|c_{B^{\prime}, x^{\prime}}\right|\right.$ is larger than $\left|c_{B, x}\right|$ by a factor 2.1). All these are indications that the ageing effect is a weak source of the asymmetry of the magnetic field in MCs, and we quantify this below.

More generally, Fig. 5 provides examples of the following results. First, correcting for the ageing effect reduces the asymmetry for MCs with stronger fields in the front, Figs. 5a and d, but does not remove it, as quantified by the $c_{B^{\prime}, x^{\prime}}$ values. A full asymmetry removal of the $B$ asymmetry would require an expansion rate that is fully incompatible with the observed velocity profile, while the velocity fits are very similar to the data (Fig. 1). Second, for some MCs, as in Figs. $5 \mathrm{~b}$ and c, the removal of the ageing effect instead increases the $B$ asymmetry, as quantified by the increase in $c_{B^{\prime}, x^{\prime}}$ compared to $c_{B, x}$. Finally, the ageing corrections have comparable results with the three types of expansion estimates (the colour curves are nearly superposed in Fig. 5), as expected from the results of Fig. 3 .

The behaviours shown in the examples of Fig. 5 are present in most MCs, as shown in Fig. 7, where the values of $c_{B^{\prime}, x^{\prime}}$, without ageing, are plotted as a function of the $c_{B, x}$ values. Removing the ageing effect implies that $c_{B^{\prime}, x^{\prime}}$ is typically shifted by a positive value because the large majority of points are above the diagonal $\left(c_{B^{\prime}, x^{\prime}}=c_{B, x}\right)$, as expected because most MCs are in expansion (Fig. 4). For MCs in expansion and with a stronger field at the rear $\left(c_{B, x}>0\right)$, this implies an increase in magnetic asymmetry $\left(c_{B^{\prime}, x^{\prime}}>c_{B, x}\right)$, while the opposite holds for MCs with a stronger field in the front $\left(c_{B, x}<0\right)$.

For a minority of MCs in contraction (with $\Delta V_{\text {lin }}>0$, marked with lighter colours in Fig. 7a), $c_{B^{\prime}, x^{\prime}}$ is slightly shifted by a low negative value compared to $c_{B, x}$ (points are below the black diagonal). We also notee that no MC is present in the lower right quadrant of the panels in Fig. 7, which is a consequence of a weak contraction and applies to only a few MCs. Finally, $c_{B^{\prime}, x^{\prime}}$ values are at variable distances from the diagonal, which shows that the correction of $c_{B, x}$ is of variable magnitude and independent of the original value of $c_{B, x}$.

The correction of $c_{B, x}$ for the ageing effect is weakly dependent on the type of velocity fit because the blue, red, and green points almost overlap in Fig. 7. These similarities are even

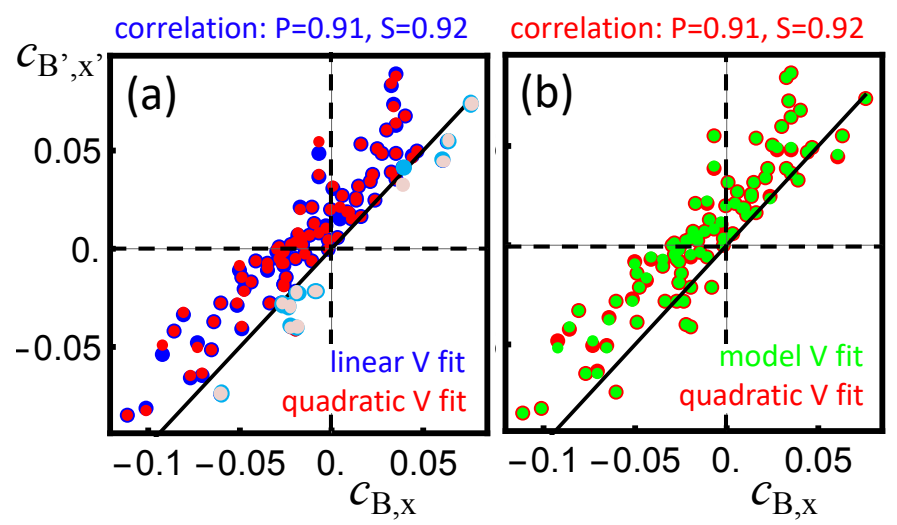

Fig. 7. Comparison of the asymmetry coefficient $c_{B, x}$ without the ageing effect (ordinate) as a function of $c_{B, x}$ derived from the uncorrected magnetic data (abscissa). (a) The results of linear and quadratic fits of the observed velocity are very similar. The points with a lighter colour are in compression $\left(\Delta V_{\operatorname{lin}}>0\right.$, see Fig. 2a). (b) The results of the model fit, Eq. (21), of the observed velocity are almost the same as the results with the quadratic fit. The diagonal black line is $c_{B^{\prime}, x^{\prime}}=c_{B, x}$. The Pearson (P) and Spearman (S) correlation coefficients are reported at the top of the panel for linear and quadratic velocity fits.

stronger than in Fig. 6. This figure shows the most extreme expansion factors. The difference of $f(t)$ between the three types of velocity fits is lower in the MC core (Fig. 3). Moreover, $f_{\text {fit }}(t)$ is the product of the contributions from the linear and quadratic terms, as shown in Eq. (16). This implies that the quadratic contribution to $f_{\text {fit }}(t)$ is the same in the MC front and rear for the same time difference with the MC centre. We conclude that the quadratic term of the velocity fit similarly corrects the two MC sides, therefore it has a weak effect on the asymmetry, and in particular on $c_{B^{\prime}, x^{\prime}}$. This implies that the global effect of removing the ageing effect on $B(x)$, as computed by $c_{B^{\prime}, x^{\prime}}$, is comparable for the three types of velocity fits.

Most importantly, Fig. 7 shows that in general, removing the ageing effect in MCs does not bring the magnetic field into a more symmetric configuration (i.e. $c_{B^{\prime}, x^{\prime}}$ closer to 0 ). The $c_{B^{\prime}, x^{\prime}}$ values are instead very closely correlated with the original $c_{B, x}$ values, as shown with the Pearson and Spearman correlation coefficients (top of Fig. 7). We conclude that the ageing effect in general contributes little to the MC asymmetry, except for a cluster of points near $c_{B^{\prime}, x^{\prime}}=0$.

\subsection{Distribution of the magnetic asymmetry}

The histograms of Fig. 8 confirm the previous results. First, the transformation of abscissa from $t$ to $x$ changes the asymmetry only weakly (Figs. 8a,b), in agreement with the earlier results from Eqs. (25) and (26). We note that all $c_{\mathrm{B}}$ values reported in Lanabere et al. (2020) are larger by a factor 2 . This rescaling does not change any of their conclusions. Next, the correction of the ageing effect globally shifts $c_{B^{\prime}, x^{\prime}}$ to slightly more positive values than $c_{B, x}$, while this does not significantly decrease the values of $\left|c_{B^{\prime}, x^{\prime}}\right|$ in general (similar standard deviation $\sigma$, and similar distribution shape as quantified by the skewness and the kurtosis). Moreover, the results are robust because the $c_{B^{\prime}, x^{\prime}}$ distribution is only weakly affected by the method we used to remove the ageing effect (Figs. 8c and d), and the histogram of the model $\mathrm{V}$ fit is similar to the quadratic fit.

The histograms also reveal points that did not stand out in Fig. 7, as follows. The distribution of $c_{B, x}$ is peaked around 

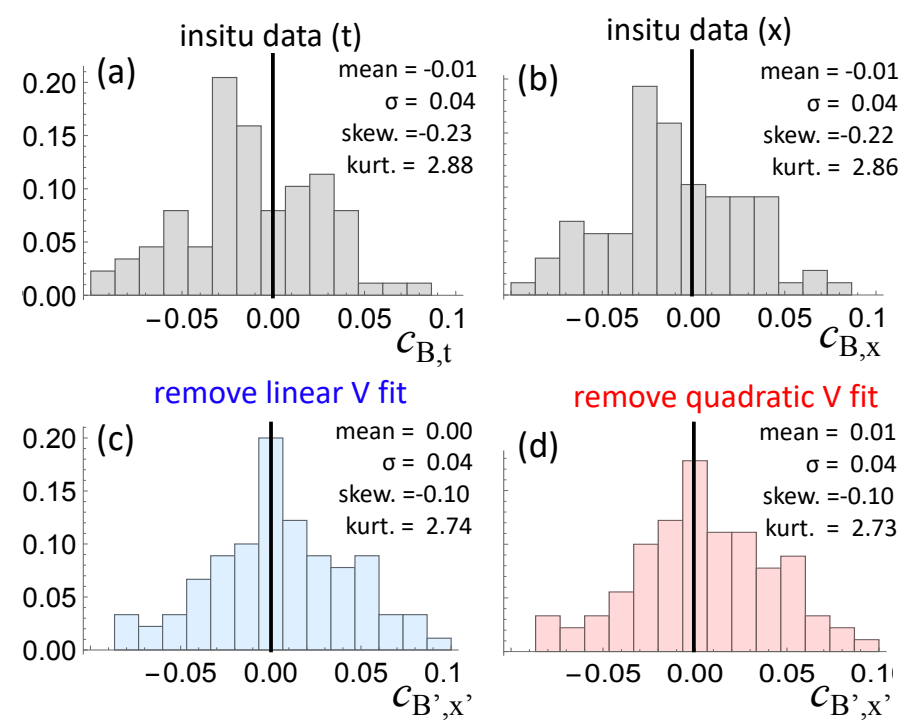

Fig. 8. Comparison of the histograms of the asymmetry coefficients. $(a, b) c_{\mathrm{B}}$ is computed directly with the magnetic data function of $(a)$ the time $t$ and $(b)$ the spatial coordinate $x$ (Eq. (2), $x$ includes the ageing effect). $(c, d) c_{B^{\prime}, x^{\prime}}$ is computed after the ageing effect is removed from the spatial coordinate ( $x^{\prime}$, Eq. (27)) and the magnetic field (Eq. (28)). The expansion factor, $f(t)$, is derived with $(c)$ a linear fit, and $(d)$ a quadratic fit of the observed velocity (Eq. (15)).

$\approx-0.025$, Figs. 8a and $\mathrm{b}$ (although the kurtosis is comparable to the value of 3 for a normal distribution). Removing the ageing effects strengthens and shifts this peak to $c_{B^{\prime}, x^{\prime}} \approx 0$. (Figs. $8 \mathrm{c}$ and d). This outstanding peak represents globally symmetric $B(x)$ profiles. Twenty-eight percent of the MCs have $\left|c_{B^{\prime}, x^{\prime}}\right|<0.01$. These MCs are expected to be closer to theoretical FR models, which are typically symmetric. For this subset of MCs, the magnetic asymmetry in observations is therefore mostly the result of the ageing effect.

The distribution of $c_{B^{\prime}, x^{\prime}}$, corrected for the ageing effect, is almost symmetric with both low mean and skewness. This is a surprising result because the physical mechanisms creating $c_{B^{\prime}, x^{\prime}}<0$ or $c_{B^{\prime}, x^{\prime}}>0$ are expected to be different. For example, a stronger total pressure in the MC sheath is expected to imply $c_{B^{\prime}, x^{\prime}}<0$, while a fast overtaking stream at the MC rear is expected to imply $c_{B^{\prime}, x^{\prime}}>0$. Next, the bending of the FR axis, concave towards the Sun, is expected to increase $B$ at the rear of the MC compared to its front, then to increase $c_{B^{\prime}, x^{\prime}}$. All these mechanisms, as well as their magnitudes, are expected to be independent, therefore they are expected to contribute differently to the magnetic field asymmetry. This means that an asymmetric distribution of $c_{B^{\prime}, x^{\prime}}$ is rather expected, in contrast with the results in Fig. 8.

\section{Conclusion}

Based on data from coronagraphic and heliospheric imagers, ICMEs are generally observed to expand when they move away from the Sun, while in situ observations confirm this with direct measurements of the proton bulk velocity. However, in contrast to imagers, in situ observations are made at various times during the spacecraft crossing, coupling spatial shape with time evolution, so that the measurements are directly affected by the ageing effect. The main aim of this study was to estimate this so-called ageing effect, then to correspondly correct the measurements in order to provide data as if they were observed at the same time along the full crossed structure. We applied the method we developed to MCs of quality 1 and 2 in Lepping's list in order to study only the best-observed cases (crossing closer to the FR core, stronger magnetic field, and less perturbed cases). This selection was expected to provide the clearest results.

The measured in situ velocity along the spacecraft trajectory was decomposed in a global and an expansion contributions. We justified that the global velocity is almost constant during the crossing of an ICME at 1 au (i.e. its limited change cannot explain the observed in situ variations of the velocity). Then, with the hypothesis of self-similar expansion during the spacecraft crossing, we derived a generic relation that expresses the expansion factor as a function of the observed velocity. With the observed duration of MCs, we showed that a Taylor expansion of the velocity up to the second order is sufficient for applications to MC data. Then, the observed velocity was fitted with either a linear or a quadratic function of time to filter the local fluctuations. Finally, the corresponding expansion factors, as a function of time, were derived along the spacecraft trajectory. We also derived the expansion factor with a model that assumed a power-law evolution of the MC size with solar distance, and the free parameters were determined by a fit to the velocity data.

The spatial coordinate $x$ along the spacecraft trajectory was computed by a temporal integration of the observed velocity. This converts time into space for each parcel of plasma and therefore adds the sizes of plasma blobs observed at different times. Then, $x$ is not a true spatial coordinate at a given time because it includes the expansion of the configuration. The derived expansion factor allowed us to correct $x$ for the ageing effect, to derive the coordinate $x^{\prime}$, Eq. (27), as if the entire MC was observed at the same time, which we set at the observed central time. Next, we corrected the magnetic field components with Eq. (28), which provided $\boldsymbol{B}^{\prime}\left(x^{\prime}\right)$. Both corrections assumed a self-similar expansion of the MC. Then, this study allowed us to both quantify the strength of the ageing effect and to remove it from the observed $\boldsymbol{B}(t)$ profile to finally deduce the spatial $\boldsymbol{B}^{\prime}\left(x^{\prime}\right)$ variations that would be produced if the observations had been made at the same time within the $\mathrm{MC}$, that is, without the ageing effect.

The shapes of the $B(t), B(x)$, and $B^{\prime}\left(x^{\prime}\right)$ profiles are quantified with the asymmetry parameters $c_{B, t}, c_{B, x}$, and $c_{B^{\prime}, x^{\prime}}$, respectively (defined by Eqs. (23) and (24)). The values $c_{B, t}$ and $c_{B, x}$ in MCs are similar, and their histograms show a shift to negative values that on average reflect stronger $B$ values in the front region of MCs. The histogram of $c_{B, x}$ is slightly transformed into that of $c_{B^{\prime}, x^{\prime}}$ when the ageing effect is removed, with very similar results for the three types of velocity profiles fitted to the data. The main change is the presence of a strong peak around $c_{B^{\prime}, x^{\prime}}=0$, that is, globally symmetric $B^{\prime}\left(x^{\prime}\right)$ profiles (Fig. 8). For this subset of MCs, about one-fourth of the studied set, the ageing effect is the main source of the observed $B(t)$ asymmetry. Next, for the fraction of MCs (about $22 \%$ ) that are in expansion and have stronger fields at the rear $\left(c_{B, x}>0\right)$, removing the ageing effect instead increases the asymmetry. For the remaining MCs $\left(c_{B^{\prime}, x^{\prime}} \lesssim 0.03\right)$, removing the ageing effect leads to magnetic profiles that are slightly more symmetric. Still, a global symmetric $B\left(c_{B^{\prime}, x^{\prime}} \approx 0\right)$ would require a far stronger expansion rate, and so it is incompatible with the observed velocity profile.

In summary, removing the ageing effect does not bring $\left|c_{B^{\prime}, x^{\prime}}\right|$ closer in general to zero than $\left|c_{B, x}\right|$ because the dispersion and the wings of the $c_{B, x}$ and $c_{B^{\prime}, x^{\prime}}$ histograms are similar. We conclude that the ageing effect is not the main origin of the observed $B(t)$ asymmetry for in situ data of MCs. Several sources of intrinsic magnetic asymmetry are possible, in particular, a higher 
compression by the surrounding medium on one MC side, either by a strong sheath at the front or by an overtaking fast stream at the rear.

Finally, while the ageing effect is typically weak, it is still worthwhile to correct for its effects, in particular, in large events (where the effects are stronger). This decouples the time evolution from the spatial magnetic configuration of MCs. We compared three types of fits of the observed velocity (one linear, one quadratic, and one derived from a power-law model for the size evolution with solar distance). They imply nearly identical corrections. This shows that the ageing effect might be well removed from any of the methods used here. These methods provide a spatial profile of $\boldsymbol{B}(x)$ that is similar to the profile that would be obtained if the full MC were observed at the same time as its centre.

Removing the ageing effect from the data is a promising alternative to techniques that fit both the velocity and magnetic data with an expanding FR model. First, no ad hoc coefficient is required to include both the magnetic field and velocity data in the minimised function. Second, the number of free parameters is decreased, which means that more elaborated magnetic models with more free parameters could be used. Third, the corrected magnetic data can be directly fitted by any static model or be analysed by an alternative method (e.g. with minimum variance analysis or by solving the Grad-Shafranov equation). This allows us to more directly compare the results derived from several methods. Finally, the method we developed to remove the ageing effect can be applied more generally to magnetic ejecta within ICMEs, assuming that they have an isotropic self-similar evolution during the spacecraft crossing.

Acknowledgements. We recognise the collaborative and open nature of knowledge creation and dissemination, under the control of the academic community as expressed by Camille Noûs at http://www. cogitamus. fr/indexen . html and we thank Bojan Vršnak for his comments which improved the manuscript. S.D. acknowledges partial support from the Argentinian grants UBACyT (UBA), and PIP-CONICET-11220130100439CO. S.D. thanks the LIA project (LIA1208). This work was partially supported by a one-month invitation of P.D. to the Instituto de Astronomía y Física del Espacio, and by a one-month invitation of S.D. to the Observatoire de Paris. This work was supported by the Programme National PNST of CNRS/INSU co-funded by CNES and CEA. S.D. is member of the Carrera del Investigador Científico, CONICET.

\section{References}

Bothmer, V., \& Schwenn, R. 1998, Ann. Geophys., 16, 1

Burlaga, L., Sittler, E., Mariani, F., \& Schwenn, R. 1981, J. Geophys. Res., 86, 6673

Cane, H. V., \& Richardson, I. G. 2003, J. Geophys. Res., 108, 1156

Cane, H. V., Richardson, I. G., \& Wibberenz, G. 1997, J. Geophys. Res., 102, 7075

Cargill, P. J., \& Schmidt, J. M. 2002, Ann. Geophys., 20, 879

Chen, J. 2017, Phys. Plasmas, 24, 090501

Dasso, S. 2009, IAU Symposium, eds. N. Gopalswamy, \& D. F. Webb, 257, 379

Dasso, S., Nakwacki, M. S., Démoulin, P., \& Mandrini, C. H. 2007, Sol. Phys., 244,115

Démoulin, P. 2010, in Twelfth International Solar Wind Conference, eds. M Maksimovic, K. Issautier, N. Meyer-Vernet, M. Moncuquet, \& F. Pantellini, Am. Inst. Phys. Conf. Ser., 1216, 329

Démoulin, P., \& Dasso, S. 2009, A\&A, 498, 551

Démoulin, P., Nakwacki, M. S., Dasso, S., \& Mandrini, C. H. 2008, Sol Phys., 250,347
Farrugia, C. J., Burlaga, L. F., Osherovich, V. A., et al. 1993, J. Geophys. Res., 98,7621

Good, S. W., Kilpua, E. K. J., LaMoury, A. T., et al. 2019, J. Geophys. Res., 124, 4960

Gosling, J. T. 1990, Am. Geophys. Union Geophys. Monograph Ser., 58, 343

Gulisano, A. M., Démoulin, P., Dasso, S., Ruiz, M. E., \& Marsch, E. 2010, A\&A, 509, A39

Gulisano, A. M., Démoulin, P., Dasso, S., \& Rodriguez, L. 2012, A\&A, 543, A107

Harrison, R. A., Davies, J. A., Rouillard, A. P., et al. 2009, Sol. Phys., 256, 219

Howard, T. A. 2011, J. Atmos. Sol. Terr. Phys., 73, 1242

Janvier, M., Winslow, R. M., Good, S., et al. 2019, J. Geophys. Res., 124, 812

Jian, L., Russell, C. T., Luhmann, J. G., \& Skoug, R. M. 2008, Adv. Space Res., 41, 259

Kilpua, E., Koskinen, H. E. J., \& Pulkkinen, T. I. 2017, Liv. Rev. Sol. Phys., 14, 5

Kumar, A., \& Rust, D. M. 1996, J. Geophys. Res., 101, 15667

Lanabere, V., Dasso, S., Démoulin, P., et al. 2020, A\&A, 635, A85

Leitner, M., Farrugia, C. J., Möstl, C., et al. 2007, J. Geophys. Res., 112, A06113

Lepping, R. P., \& Wu, C. C. 2010, Ann. Geophys., 28, 1539

Lepping, R. P., Burlaga, L. F., \& Jones, J. A. 1990, J. Geophys. Res., 95, 11957

Lepping, R. P., Berdichevsky, D. B., Szabo, A., Arqueros, C., \& Lazarus, A. J. 2003, Sol. Phys., 212

Lepping, R. P., Wu, C. C., Berdichevsky, D. B., \& Ferguson, T. 2008, Ann. Geophys., 26, 1919

Lepping, R. P., Wu, C. C., Berdichevsky, D. B., \& Szabo, A. 2015, Sol. Phys., 290, 2265

Liu, Y., Richardson, J. D., \& Belcher, J. W. 2005, Planet. Space Sci., 53, 3

Liu, Y. D., Hu, H., Wang, C., et al. 2016, ApJS, 222, 23

Lugaz, N., Manchester, W. B., IV, \& Gombosi, T. I. 2005, ApJ, 627, 1019

Lynch, B. J., Zurbuchen, T. H., Fisk, L. A., \& Antiochos, S. K. 2003, J. Geophys. Res., 108, A01239

Lynnyk, A., \& Vandas, M. 2009, Planet. Space Sci., 57, 1375

Manchester, W. B., Gombosi, T. I., Roussev, I., et al. 2004, J. Geophys. Res., 109,2107

Marubashi, K., \& Lepping, R. P. 2007, Ann. Geophys., 25, 2453

Marubashi, K., Cho, K.-S., \& Ishibashi, H. 2017, Sol. Phys., 292, 189

Masías-Meza, J. J., Dasso, S., Démoulin, P., Rodriguez, L., \& Janvier, M. 2016, A\&A, 592, A118

Möstl, C., Amla, K., Hall, J. R., et al. 2014, ApJ, 787, 119

Nakwacki, M., Dasso, S., Mandrini, C. H., \& Démoulin, P. 2008, J. Atm. Sol. Terr. Phys., 70, 1318

Nakwacki, M., Dasso, S., Démoulin, P., Mandrini, C. H., \& Gulisano, A. M. 2011, A\&A, 535, A52

Osherovich, V. A., Farrugia, C. J., Burlaga, L. F., et al. 1993, J. Geophys. Res., 98, 15331

Rodriguez, L., Masías-Meza, J. J., Dasso, S., et al. 2016, Sol. Phys., 291, 2145

Rouillard, A. P. 2011, J. Atm. Sol. Terr. Phys., 73, 1201

Rust, D. M., Anderson, B. J., Andrews, M. D., et al. 2005, ApJ, 621, 524

Salman, T. M., Winslow, R., \& Lugaz, N. 2020, J. Geophys. Res., 125, e2019JA027084

Schwenn, R. 2006, Liv. Rev. Sol. Phys., 3, 2

Sheeley, N. R., Jr, Howard, R. A., Michels, D. J., et al. 1985, J. Geophys. Res., 90, 163

Shimazu, H., \& Vandas, M. 2002, Earth Planets Space, 54, 783

Vandas, M., \& Romashets, E. 2017, A\&A, 608, A118

Vandas, M., Romashets, E., \& Geranios, A. 2015, A\&A, 583, A78

Vandas, M., Romashets, E. P., Watari, S., et al. 2006, Adv. Space Res., 38, 441

Vršnak, B., Amerstorfer, T., Dumbović, M., et al. 2019, ApJ, 877, 77

Wang, C., Du, D., \& Richardson, J. D. 2005, J. Geophys. Res., 110, A10107

Wang, Y., Shen, C., Liu, R., et al. 2018, J. Geophys. Res., 123, 3238

Wimmer-Schweingruber, R. F., Crooker, N. U., Balogh, A., et al. 2006, Space Sci. Rev., 123, 177

Winslow, R. M., Lugaz, N., Philpott, L. C., et al. 2015, J. Geophys. Res., 120, 6101

Wood, B. E., Wu, C.-C., Lepping, R. P., et al. 2017, ApJS, 229, 29 Xiong, M., Zheng, H., Wang, Y., \& Wang, S. 2006, J. Geophys. Res., 111,

Zhao, X., Liu, Y. D., Hu, H., \& Wang, R. 2019, ApJ, 882, 122

Zurbuchen, T. H., \& Richardson, I. G. 2006, Space Sci. Rev., 123, 31 